Preface

\title{
Hemostasis and Thrombosis in Extreme Physiological and Pathological Conditions
}

\author{
Benjamin Brenner, $\mathrm{MD}^{2,3}$ Ton Lisman, $\mathrm{PhD}^{1}$ \\ 1 Section of Hepatobiliary Surgery and Liver Transplantation, \\ Department of Surgery, University Medical Center Groningen, \\ University of Groningen, Groningen, The Netherlands \\ 2 Department of Hematology and Bone Marrow Transplantation, \\ Rambam Health Care Campus, Haifa, Israel \\ 3 Ruth and Bruce Rappaport Faculty of Medicine, Technion, Haifa, Israel \\ Semin Thromb Hemost 2018;44:615-616.
}

The hemostatic system is responsible for limiting blood loss upon vascular injury and is designed to rapidly react to breaches in the endothelial cell lining. Components of this system are subject to powerful regulatory mechanisms including various antihemostatic actions of the endothelium, antihemostatic actions from proteins in plasma, and blood flow that removes activated hemostatic proteins from the growing thrombus. A well-orchestrated balance between pro- and antihemostatic forces ensures hemostatic balance and avoids spontaneous bleeding or thrombotic episodes. It is evident that hemostatic balance is crucial to avoid these complications, as evidenced by the pathology associated with a deficiency of pro- or anticoagulant proteins (e.g., severe and spontaneous bleeding in hemophilia and substantially elevated thrombotic risk in patients with deficiencies in some of the natural anticoagulants). While congenital or acquired disorders may alter multiple components of the hemostatic system simultaneously, such complex changes in the hemostatic system may not necessarily lead to severe pathology. For example, the complex hemostatic changes in patients with liver disease lead to a hemostatic rebalance without a clinical phenotype in many patients, which demonstrates the resilience of the hemostatic system. ${ }^{1}$ Likewise, hemostatic challenges resulting from surgery or trauma are not necessarily related to a dismal hemostatic outcome. $^{2,3}$ In this issue of Seminars of Thrombosis and Hemostasis, we explored consequences of remarkable physiological or pathological conditions on the hemostatic system. As we will see, some "extreme" conditions may be without clinical consequences, whereas others are related to a substantial risk of bleeding or thrombosis. Moreover, there is a remarkable set of challenges to the hemostatic system, that is, hemostatic activators or inhibitors in a variety of venoms, which are potentially useful as therapeutic agents.

We will start this issue by discussing effects of physiological extremes. First, Kenet et $\mathrm{al}^{4}$ discuss the hemostatic system in the very young. It is well known that the hemostatic system in newborns and very young children has a remarkably different composition than that in adults, with decreased plasma levels of most of the activators and inhibitors. Despite these differences, most children fortunately do well and are in excellent hemostatic balance. The paper by Kenet et al discusses those rare cases in which young children suffer from bleeding or thrombotic complications in the context of the concept of developmental hemostasis. Next, Tzoran et $\mathrm{al}^{5}$ discuss the hemostatic changes that occur as a consequence of aging. As in the very young, the hemostatic system in the oldest old is substantially different from younger adults. However, in this population, the risk of thrombotic disease increases tremendously, and interactions with chronic illnesses are associated with important thrombotic issues. The subsequent "extreme condition" is discussed by Hunt, ${ }^{6}$ who addresses hemostatic changes in extremes of body weight. Very low body weight is associated with incompletely characterized hemostatic changes, which include decreased blood cell counts and platelet hyperaggregability. Among the many clinical consequences of severe obesity is a hypercoagulable state that is related to an increased risk of venous and arterial thrombosis. The author also discusses mechanisms and clinical consequences of the hemostatic changes associated with (severe) obesity. The next paper by Kicken et $\mathrm{al}^{7}$ deals with extreme exertion. The authors propose that a hypercoagulable state induced by strenuous exercise is balanced by a concomitant

\author{
Address for correspondence \\ Ton Lisman, PhD, Section of \\ Hepatobiliary Surgery and Liver \\ Transplantation, Department of \\ Surgery, University Medical \\ Center Groningen, University of \\ Groningen, BA33, Hanzeplein 1, \\ 9713 GZ Groningen, \\ The Netherlands \\ (e-mail: j.a.lisman@umcg.nl).
}

Issue Theme Hemostasis and Thrombosis in Extreme Physiological and Pathological Conditions; Guest Editors: Benjamin Brenner, MD, and Ton Lisman, PhD.
Copyright $\odot 2018$ by Thieme Medical Publishers, Inc., 333 Seventh Avenue, New York, NY 10001, USA. Tel: +1(212) 584-4662.
DOI https://doi.org/ 10.1055/s-0038-1666826. ISSN 0094-6176. 
development of a hyperfibrinolytic state. However, this compensatory mechanism largely disappears during recovery, which may be important in the context of an increased risk of cardiovascular events in athletes.

The next section in this issue discusses hemostatic challenges caused by disease. Levi ${ }^{8}$ discusses effects of abnormal body temperature on the hemostatic system. Heatstroke is used to provide an example of hemostatic changes that may occur as a result of hyperthermia. Heatstroke may lead to disseminated intravascular coagulation, which may contribute to a dismal outcome. Hypothermia may occur in conditions such as trauma or imposed intentionally to protect the brain in patients who have had a cardiac arrest or acute liver failure. Although hemostatic changes do occur, the clinical consequences may be limited. Hoirisch-Clapauch ${ }^{9}$ then discusses the risks of bleeding and thrombosis related to anxiety. The author puts forward the thought-provoking hypothesis that anxiety combined with hypofibrinolytic states (defined as severe depression or a high-carbohydrate, low-protein, low-fiber diet and a sedentary lifestyle) has a role in the pathogenesis of thrombotic events and that part of these events, currently classified as unprovoked, are in reality provoked by high anxiety states. The next paper by Bentur et $\mathrm{al}^{10}$ deals with the effects of stress on the hemostatic system and concludes that the hypercoagulable state induced by stress could be related to a combination of neurotransmitters and hormones, and the effects of stress on hemodynamics. Vadasz and Toubi ${ }^{11}$ then discuss the hemostatic changes in allergy, specifically in asthma and chronic spontaneous urticaria. The authors show complex hemostatic changes in both disorders and demonstrate that these changes are, at least partly, related to the effects of the immune system on hemostasis. Subsequently, Elbers et al ${ }^{12}$ discuss the interplay between thyroid hormones and hemostasis, showing that hypothyroidism is associated with hypocoagulability and risk of bleeding, and hyperthyroidism is associated with hypercoagulability and risk of venous thrombosis. In addition, the paper describes effects of malignant thyroid disease on developing venous thrombosis, which relates to local stasis of the blood due to compression effects of the expanding tumor. The next paper by Putri et $\mathrm{al}^{13}$ discusses thrombocytopenia and platelet function defects that are frequent complications of tropical diseases such as malaria and Dengue fever. Interestingly, in some tropical diseases in which patients commonly develop thrombocytopenia bleeding is rare (e.g., in malaria), whereas in other diseases, bleeding is an important complication of the disease, which may be related to preservation of platelet function in malaria. The paper therefore discusses mechanisms and clinical consequences of thrombocytopenia and platelet function defects in tropical diseases and highlights the difficulties in studying platelet properties in resource-poor countries where these such diseases are frequent. A final contribution by Koh et $\mathrm{al}^{14}$ gives an extensive overview of proteins and peptides isolated from different types of venoms that interfere with a variety of hemostatic processes. The paper also gives an overview of discovery and development of venom-derived antiplatelet, anticoagulant, and thrombolytic agents. Venom-derived compounds are not only used in diagnostic assays of hemostasis (e.g., ristocetin-induced platelet agglutination or the Russell Viper Venom time) but have clear potential as pharmacological agents (e.g., hirudin).

Taken together, the papers in this issue of the journal highlight subtle or pronounced hemostatic effects of a wide variety of physiological or pathological extremes. The clinical consequences associated with hemostatic alterations by these extremes may teach us valuable lessons about the requirements for hemostatic balance in health and disease. In addition, these reviews accentuate the variety of triggers that may alter the hemostatic system, and stress links between physiological or pathological conditions and hemostasis that may not be immediately apparent. We sincerely hope you enjoy reading the wide range of papers assembled in this issue.

\section{References}

1 Lisman T, Porte RJ. Pathogenesis, prevention, and management of bleeding and thrombosis in patients with liver diseases. Res Pract Thromb Haemost 2017;1(02):150-161

2 Hvas AM, Larsen JB, Pasalic L. Thrombosis and hemostasis in surgery. Semin Thromb Hemost 2017;43(07):649-652

3 Harris T, Davenport R, Mak M, Brohi K. The evolving science of trauma resuscitation. Emerg Med Clin North Am 2018;36(01): 85-106

4 Kenet G, Barg AA, Nowak-Göttl U. Hemostasis in the very young. Semin Thromb Hemost 2018;44(07):617-623

5 Tzoran I, Hoffman R, Monreal M. Hemostasis and thrombosis in the oldest old. Semin Thromb Hemost 2018;44(07):624-631

6 Hunt BJ. Haemostasis at extremes of body weight. Semin Thromb Hemost 2018;44(07):632-639

7 Kicken $\mathrm{CH}$, Miszta A, Kelchtermans H, de Laat B. Hemostasis during extreme exertion. Semin Thromb Hemost 2018;44(07): 640-650

8 Levi M. Hemostasis and thrombosis in extreme temperatures (hypo- and hyperthermia). Semin Thromb Hemost 2018;44(07): 651-655

9 Hoirisch-Clapauch S. Anxiety related bleeding and thrombosis. Semin Thromb Hemost 2018;44(07):656-661

10 Bentur OS, Sarig G, Brenner B, Jacob G. Effects of acute stress on thrombosis. Semin Thromb Hemost 2018;44(07):662-668

11 Vadasz Z, Toubi E. Hemostasis in allergy. Semin Thromb Hemost 2018;44(07):669-675

12 Elbers LP, Squizzato A, Gerdes VE. Thyroid disorders and hemostasis. Semin Thromb Hemost 2018;44(07):676-682

13 Putri IH, Tunjungputri RN, de Groot PG, van der Ven AJ, de Mast Q. Thrombocytopenia and platelet dysfunction in acute tropical infectious diseases. Semin Thromb Hemost 2018;44(07): 683-690

14 Koh CY, Modahl CM, Kulkarni N, Kini RM. Toxins are an excellent source of therapeutic agents against cardiovascular diseases. Semin Thromb Hemost 2018;44(07):691-706 